\title{
INTERSECTIONS OF COMMUTANTS WITH CLOSURES OF DERIVATION RANGES
}

\author{
DOMINGO A. HERRERO
}

\begin{abstract}
The norm closure of the set $\mathbb{Q}_{w}(\mathcal{X})=\bigcup\left\{\operatorname{Ran}\left(\delta_{A}\right)^{-w} \cap\{A\}^{\prime}\right.$ : $A \in \mathcal{L}(X)\}$, where $\delta_{A}$ denotes the inner derivation induced by the operator $A, \operatorname{Ran}\left(\delta_{A}\right)^{-w}$ is the weak closure of the range of $\delta_{A}$ and $\{A\}^{\prime}$ is the commutant of $A$, is disjoint from the open dense subset $\mathscr{B}(\mathscr{X})=\{T \in$ $\mathfrak{L}(\mathscr{X}): T$ has a nonzero normal eigenvalue $\}$ for every complex Banach space $\mathcal{X}$. For a Hilbert space $\mathcal{X}, \mathcal{L}(\mathcal{H})=\mathscr{B}(\mathscr{X}) \cup \mathbb{A}_{w}(\mathcal{X})^{-}$, where the bar denotes norm closure.
\end{abstract}

1. Introduction. Let $\mathfrak{X}$ be a (nonzero) complex Banach space and let $\mathcal{L}(\mathfrak{X})$ denote the algebra of all (bounded linear) operators acting on $\mathcal{X}$. To each $\mathscr{Q} \in \mathcal{L}(\mathscr{X})$ we associate the following objects: $\delta_{A}$ (the inner derivation of $\mathcal{L}(\mathcal{X})$ defined by $\left.\delta_{A}(X)=A X-X A\right), \operatorname{Ran}\left(\delta_{A}\right)^{-}$(the norm closure of the range of $\left.\delta_{A}\right), \operatorname{Ran}\left(\delta_{A}\right)^{-w}$ (the closure of the range of $\delta_{A}$ in the weak operator topology), $\{A\}^{\prime}$ (the commutant of $A$ ), $\sigma(A)$ (the spectrum of $A$ ) and $\operatorname{sp}(A)$ (the spectral radius of $A$ ).

In [15] Hong W. Kim analyzed the set

$$
\mathbb{Q}=\bigcup\left\{\operatorname{Ran}\left(\delta_{A}\right)^{-} \cap\{A\}^{\prime}: A \in \mathcal{L}(\mathscr{X})\right\}
$$

for the case when $\mathfrak{X}=\mathscr{H}$ is a Hilbert space and raised the following problems:

[15, Question 1]. Is $\mathfrak{L}(\mathscr{X}) \backslash \mathfrak{Q}$ (norm) dense in $\mathfrak{L}(\mathfrak{X})$ ?

[15, Question 3]. Is every thin operator in $Q$ ?

(where $B \in \mathcal{L}(\mathfrak{X})$ is thin, in the sense of A. Brown and C. Pearcy [4], if $B=\lambda-K$ for some $\lambda \in \mathbf{C}=$ complex plane, identified with the multiples of the identity operator, and some $K \in \mathscr{K}=$ the ideal of compact operators). The subset of all thin operators will be denoted by $(T)$.

The answer to the first question is yes. Indeed, more is actually true: $\mathbb{Q}_{w}=\bigcup\left\{\operatorname{Ran}\left(\delta_{A}\right)^{-w} \cap\{A\}^{\prime}: A \in \mathcal{L}(\mathcal{X})\right\}$ is nowhere dense in $\mathcal{L}(\mathfrak{X})$ for every complex Banach space $\mathcal{X}$.

The answer to the third question is no: If $\lambda-K \in \mathbb{Q}_{w}^{-}$, then $\sigma(\lambda-K) \subset$ $\{0, \lambda\}$ (for every $\mathfrak{X}$ as well).

Received by the editors May 9, 1978.

AMS (MOS) subject classifications (1970). Primary 16A72, 47B47.

Key words and phrases. Inner derivations, norm closure, weak closure, weak* closure, closure of the range of an inner derivation, commutant, normal eigenvalue, biquasitriangular operators. 
Recall that a point $\lambda \in \sigma(T)$ is a normal eigenvalue of $T$ if $\lambda$ is isolated in $\sigma(T)$ and $\mathcal{X}=\mathscr{X}_{\lambda} \oplus \mathcal{X}_{\lambda}^{\prime}$, where $\mathfrak{X}_{\lambda}, \mathscr{X}_{\lambda}^{\prime}$ are the spectral invariant subspaces of $T$ such that $\sigma\left(T_{\lambda}\right)=\{\lambda\}\left(T_{\lambda}=T \mid \mathscr{X}_{\lambda}\right.$ is the restriction of $T$ to $\left.\mathscr{X}_{\lambda}\right)$ and $\lambda \notin \sigma\left(T \mid \mathscr{X}_{\lambda}^{\prime}\right)$, and $\mathcal{X}_{\lambda}$ is finite dimensional. Then we have

THEOREM 1. $\mathbb{Q}_{w}^{-}$is disjoint from the open dense set $\mathscr{B}=\{T \in \mathcal{L}(\mathcal{X})$ : $\left.\sigma_{0}(T) \cap[\mathbf{C} \backslash\{0\}] \neq \varnothing\right\}$, where $\sigma_{0}(T)$ denotes the set of all normal eigenvalues of $T$.

In particular, if $B=\lambda-K \in \mathbb{Q}_{w}^{-} \cap(T)$, then either $K$ is a compact quasinilpotent operator or $\sigma(K)=\{0, \lambda\}$. $Q_{w}^{-} \cap(T)$ is nowhere dense in $(T)$.

Proof. That $\mathscr{B}$ is open and dense and, similarly, that $\mathscr{B} \cap(T)$ is open and dense in $(T)$ follows from [13]. Thus, in order to complete the proof, it suffices to show that $Q_{w} \cap \mathscr{B}=\varnothing$, and this follows from [16, Theorem 3]. (Indeed, the assumption that $\mathcal{H}$ is a Hilbert space is irrelevant in [16]: no modifications are necessary for the case of an arbitrary Banach space $\mathfrak{X}$.)

For a Hilbert space $\mathcal{H}$, Theorem 1 gives the best possible answer. Recall that the weak* (or ultraweak) topology of $\mathcal{L}(\mathcal{X})$ (i.e., the weak* topology as the dual of the Banach space of all trace class operators; see [5, p. 39]) is strictly stronger than the weak operator topology. Hence,

$$
\mathbb{Q}_{w^{*}}=\bigcup\left\{\operatorname{Ran}\left(\delta_{A}\right)^{-w^{*}} \cap\{A\}^{\prime}: A \in \mathcal{L}(\mathcal{H})\right\}
$$

is a subset of $\mathbb{Q}_{w}$ and therefore, by Theorem 1 ,

$$
\left(\mathbb{Q}_{w^{*}}\right)^{-} \subset \mathbb{Q}_{w}^{-} \subset \mathcal{L}(\mathcal{H}) \backslash \mathscr{B} .
$$

These inclusions are actually equalities; in fact, we have

THEOREM 2. For an infinite dimensional Hilbert space $\mathcal{H}, \mathcal{L}(\mathcal{H})$ is the disjoint union of $\mathscr{B}$ and $\left(\mathbb{Q}_{w^{*}}\right)^{-}$, i.e., $\left(\mathbb{Q}_{w^{*}}\right)^{-}=\mathbb{Q}_{w}^{-}=\mathscr{L}(\mathcal{H}) \backslash \mathscr{B}$.

The proof of this theorem will be given in $\S 3$. The author wishes to thank Professor L. A. Fialkow for providing useful references.

\section{Complementary results.}

LemMA 1. (i) $\mathbb{Q}\left(\mathbf{C}^{n}\right)=\mathscr{Q}\left(\mathbf{C}^{n}\right)^{-}=\left\{Q \in \mathcal{L}\left(\mathbf{C}^{n}\right): Q\right.$ is nilpotent $\}, n=1,2$, $3, \ldots$.

(ii) For every $\mathcal{X}, \mathcal{Q}$ contains every finite rank nilpotent operator.

Proof. Let $Q_{k}, H_{k} \in \mathcal{L}\left(\mathbf{C}^{k}\right)$ be the operators defined by $Q_{k} e_{1}=0, Q_{k} e_{j}=$ $e_{j-1}, j=2,3, \ldots, k$, and $H_{k} e_{j}=j e_{j}, j=1,2,3, \ldots, k$, where $\left\{e_{j}\right\}_{j=1}^{k}$ is the canonical basis of $\mathbf{C}^{k}$. Then (as in [6, Chapter 19])

$$
Q_{k} H_{k}-H_{k} Q_{k}=Q_{k} \in \operatorname{Ran}\left(\delta_{Q_{k}}\right) \cap\left\{Q_{k}\right\}^{\prime} .
$$

By considering finite direct sums and Jordan forms, we obtain (i); (ii) is a trivial consequence of (i) and the Hahn-Banach theorem. 
LEMMA 2. Let $T \in \mathcal{L}(\mathcal{X})$ and assume that there exists an idempotent $P$ such that $P A=A P$ for all $A \in\{T\}^{\prime}$. Then $T \in \mathbb{Q}(\mathscr{X})$ if and only if $T \mid \operatorname{Ran} P \in$ $\mathcal{Q}(\operatorname{Ran} P)$ and $T \mid \operatorname{Ker} P \in \mathbb{Q}(\operatorname{Ker} P)$.

In particular, if $\sigma(T)$ is disconnected, then $T \in \mathcal{Q}(\mathcal{X})$ if and only if $T \mid \mathcal{X}_{\sigma} \in$ $\mathbb{Q}\left(\mathfrak{X}_{\sigma}\right)$ for every spectral invariant subspace $\mathfrak{X}_{\sigma}$ (associated with a clopen subset $\sigma$ of $\sigma(T)$ via Riesz decomposition theorem).

The proof of the first part is straightforward. For the second part it is enough to recall that, if $P_{\sigma}$ is the projection of $\mathcal{X}$ onto $\mathcal{X}_{\sigma}$ along its complementary spectral invariant subspace, then $P_{\sigma}$ commutes with every $A \in\{T\}^{\prime}$, so that $\{T\}^{\prime}$ "splits", etc. (see, e.g., $\left.[14, \S 5]\right)$.

The following result is a minor improvement of the Theorem in [15].

LEMMA 3. If $T$ is bounded below by a positive constant $\alpha$ and

$$
\phi(A, n)=(1 / n) \sum_{i=0}^{n-1}\left(\left\|A^{n-i-1}\right\| \cdot\left\|A^{i}\right\| /\left\|A^{n-1}\right\|\right) \leqslant C(A),
$$

where $C(A)$ is a constant depending on $A$, for every $A \in\{T\}^{\prime}$ such that $\sigma(A) \subset D(2,1)=\{\lambda:|\lambda-2|<1\}$, then $T \notin \mathbb{Q}$.

Proof. Assume that $T \in \mathbb{Q}$; then

$$
\lim (j \rightarrow \infty)\left\|\delta_{A}\left(X_{j}\right)-T\right\|=0
$$

for some $A \in\{T\}^{\prime \prime}$ and a suitable sequence $\left\{X_{j}\right\}_{j=1}^{\infty} \subset \mathcal{L}(\mathcal{X})$.

Replacing, if necessary, $A$ by $2+c A$ for some $c>0$ small enough, we can directly assume that $\sigma(A) \subset D(2,1)[6$, Chapter 19]; then a trivial change of the proof of Theorem in [15] (see also [6, Problem 185]) shows that

$$
\begin{aligned}
\alpha & \leqslant(1 / n)\left\{2\|A\| \cdot\left\|X_{j}\right\|+\left\|\delta_{A}\left(X_{j}\right)-T\right\| n \phi(A, n)\right\} \\
& \leqslant\left(2\|A\| \cdot\left\|X_{j}\right\|\right) / n+C(A)\left\|\delta_{A}\left(X_{j}\right)-T\right\|
\end{aligned}
$$

for all $n, j \geqslant 1$ and, a fortiori,

$$
0<\alpha \leqslant C(A)\left\|\delta_{A}\left(X_{j}\right)-T\right\| \rightarrow 0 \quad(j \rightarrow \infty),
$$

a contradiction.

REMARK. If $A \in \mathcal{L}\left(\mathbf{C}^{k}\right)$ and $\sigma(A) \subset D(2,1)$, then $A=W J W^{-1}$ for some invertible $W$ and some Jordan form $J$ whose eigenvalues lie outside the closed unit disk $D^{-}=\{\lambda:|\lambda| \leqslant 1\}$; we obtain

$$
\operatorname{sp}(A)^{i} \leqslant\left\|A^{i}\right\| \leqslant \operatorname{sp}(A)^{i}+k\|W\| \cdot\left\|W^{-1}\right\| \operatorname{sp}(A) \leqslant \operatorname{sp}(A)^{i}\left(1+k\|W\| \cdot\left\|W^{-1}\right\|\right),
$$

whence it readily follows that $\phi(A, n)=C(A)=\left(1+k\|W\| \cdot\left\|W^{-1}\right\|\right)^{2}$.

\section{The Hilbert space case.}

Proof of Theorem 2. Assume that $T \notin \Re$; then $T$ is similar to $T_{0} \oplus T_{1}$, where $T_{0}$ is a nilpotent operator acting on a subspace $\mathcal{H}_{0}$ of finite dimension $d, 0 \leqslant d<\infty$, and $T_{1}$ acts on an infinite dimensional subspace $\mathcal{H}_{1}$ and $\sigma_{0}\left(T_{1}\right)=\varnothing$. 
Assume that $\mathcal{H}$ is separable; then it follows from the proof of $[9$, Theorem 3] that $T_{1}$ is the norm limit of a sequence $\left\{A_{n}\right\}_{n=1}^{\infty}$ in $\mathcal{L}\left(\mathcal{F}_{1}\right)$ such that $\left\{A_{n}\right\}^{\prime}=\left\{A_{n}\right\}^{\prime \prime}$ (= double commutant) does not contain any nonzero compact operator.

According to [19, Corollary 1] $\operatorname{Ran}\left(\delta_{A}\right)^{-w^{*}}=\mathcal{L}(\mathcal{H})$ if and only if $\{A\}^{\prime}$ does not contain any nonzero trace class operator. Hence, $A_{n} \in\left\{A_{n}\right\}^{\prime} \subset$ $\operatorname{Ran}\left(\delta_{A_{n}}\right)^{-w^{*}}=\mathcal{L}\left(\mathcal{G}_{1}\right)$ for all $n=1,2, \ldots$, and, a fortiori, $T_{1} \in \mathbb{Q}_{w^{*}}\left(\mathcal{H}_{1}\right)^{-}$.

By using Lemma 1(i) and the fact that $\mathcal{Q}$ (and therefore $\mathcal{Q}^{-}$too $[11, \S 2]$ ) is invariant under similarities, we conclude that $T$ belongs to $\mathbb{Q}_{w^{*}}(\mathcal{H})^{-}$.

In the nonseparable case, $T_{1}=\bigoplus_{\nu \in \Gamma} T_{1 \nu}\left(T_{1}\right.$ is unitarily equivalent to the orthogonal direct sum of the family $\left.\left\{T_{1 v}\right\}_{\nu \in \Gamma}\right)$, where $\operatorname{card}(\Gamma)=\operatorname{dim} \mathcal{H}$ and $\sigma_{0}\left(T_{1 v}\right)=\varnothing$ for all $\nu \in \Gamma$ (see, e.g., [10, Theorem 3]).

Combining this decomposition with the proof of [9, Theorem 3] we can find a sequence $\left\{A_{n}=\bigoplus_{\nu \in \Gamma} A_{1 v n}\right\}_{n=1}^{\infty}$ (the decomposition of $\mathcal{H}_{1}$ being the same as for $T_{1}$, of course) of operators in $\mathcal{L}\left(\mathcal{F}_{1}\right)$ such that $A_{1 v n}$ is similar to an operator in a finite family $\left\{B_{1}, B_{2}, \ldots, B_{r(n)}\right\},\left\{B_{j}\right\}^{\prime}=\left\{B_{j}\right\}^{\prime \prime}$ does not contain any nonzero compact operator and $\left\{A_{n}\right\}^{\prime} \cap \mathcal{K}\left(\mathcal{K}_{1}\right)=\{0\}$ for every $n=1,2,3, \ldots$ Exactly as in the separable case, we conclude that $T \in$ $\mathbb{Q}_{w^{*}}(\mathcal{H})^{-}$.

We close the paper with some remarks and open problems.

(a) If $\mathcal{H}$ is a complex separable infinite dimensional Hilbert space and $K$ is unitarily equivalent to the orthogonal direct sum of the compact operators $\left\{(1 / k) Q_{k}\right\}_{k=1}^{\infty}\left(K \cong \bigoplus_{k=1}^{\infty}(1 / k) Q_{k}\right)$, where $Q_{k}$ is the operator defined in the proof of Lemma 1(ii), then $K \in \mathcal{K}$ and the arguments used in that lemma show that $K \in \operatorname{Ran}\left(\delta_{K}\right)^{-} \cap\{K\}^{\prime}$; furthermore, if $L \cong \bigoplus_{k=1}^{\infty}(1 / k !)^{2} Q_{k}$, then $L \in \delta_{K}(\mathcal{K}) \cap\{K\}^{\prime}$. Thus, $\mathbb{Q} \mathcal{K}=\cup\left\{\operatorname{Ran}\left(\delta_{K}\right)^{-} \cap\{K\}^{\prime}: K \in \mathscr{K}\right\}$ contains a quasinilpotent operator which is not nilpotent (similar examples can be obtained for an arbitrary infinite dimensional Banach space $\mathcal{X}$ by using Markushevich bases [17]).

Combining Lemma 1(ii) with a result of R. G. Douglas (every compact quasinilpotent is a norm limit of finite rank nilpotents [7, Problem 7]), it readily follows that $\mathbb{Q}^{-} \cap \mathscr{K}=\mathbb{Q}_{w}^{-} \cap \mathscr{K}=\mathscr{Q} \mathcal{K}^{-}=\{K \in \mathscr{K}: K$ is a quasinilpotent\}.

QuEstion 1. Is $\mathbb{Q} \cap \mathcal{K}$ a proper subset of $\mathscr{Q} \mathcal{K}^{-}$? Is $\mathbb{Q} \mathcal{K}$ a proper subset of $Q \mathcal{K}^{-}$?

Question 2. Does $1-K \in \mathbb{Q}$ for some nonzero compact quasinilpotent $K$ ?

(b) By a celebrated theorem of J. H. Anderson [1], it readily follows that if $N=$ (Nilpotent acting on a finite dimensional space) $\bigoplus\left\{\bigoplus_{\lambda \in \Lambda} I_{\lambda}\right\}$, where $\Lambda$ is an arbitrary bounded subset of $\mathbf{C}$ and $I_{\lambda}$ is the identity operator on a Hilbert space $\mathcal{H}_{\lambda}$ of infinite dimension, then $N \in \mathbb{Q}$. On the other hand, if

$$
L=\int_{\sigma(T)} \lambda d E_{\lambda}
$$


is the spectral decomposition of the normal operator $L$ and there exists a Borel set $\Omega \subset \mathbf{C} \backslash\{0\}$ such that $E(\Omega) \neq 0$ and $L_{\Omega}=L \mid E(\Omega) \mathcal{H}$ has uniform finite multiplicity (the reader is referred to [8] for definitions and properties), then $L \notin \mathbb{Q}$. (Proof. $C\left(A_{\Omega}\right)=$ [multiplicity of $L_{\Omega}$ ] for all $A_{\Omega} \in\left\{L_{\Omega}\right\}^{\prime}$. Now apply Lemmas 2 and 3.) In particular, if $U$ is the bilateral shift, then $U^{(n)}=U+U+\cdots+U(n$ copies $)$ belongs to $Q^{-} \backslash \mathbb{Q}, n=1,2, \ldots$

QuEstion 3. Which normal operators belong to $\mathbb{Q}$ ? Does $U^{(\infty)}=U \oplus U$ $\oplus U \oplus \ldots$ belong to $\mathbb{Q}$ ?

(c) If $\mathcal{H}$ is separable, then $T \in \mathcal{L}(\mathcal{H})$ is biquasitriangular if ind $(\lambda-T)=0$ for all $\lambda \in \mathbf{C}$ such that $\lambda-T$ is a semi-Fredholm operator [2]; for a nonseparable $\mathcal{H}$, biquasitriangularity is defined in terms of the weighted spectra of $T$ (see [10]). Let (BQT) denote the class of all biquasitriangular operators acting in $\mathcal{H}$.

The above mentioned consequence of Anderson's theorem and the fact that $\mathbb{Q}^{-}$is invariant under similarities imply that $\delta(N)^{-} \subset \mathbb{Q}^{-}$, where $\delta(N)=\left\{W N W^{-1}: W\right.$ is invertible in $\left.\mathcal{L}(\mathcal{H})\right\}$, for every $N$ as in (b), whence we obtain the following

Corollary 1. $(B Q T) \backslash \Re \subset \mathbb{Q}^{-}$. In particular, $(T) \backslash \mathscr{B} \subset \mathbb{Q}^{-}$.

Proof. It follows from [3] and [10] (see also [12], [18]) that (BQT) \} \mathscr { B } = $\left[\cup\left\{\delta(N)^{-}: N\right.\right.$ as in $\left.\left.\left.b\right)\right\}\right]^{-}$(the outer closure is irrelevant in the separable case). Hence, $(\mathrm{BQT}) \backslash \mathscr{B} \subset \mathbb{Q}^{-}$.

Conjecture. $Q^{-}=(\mathrm{BQT}) \backslash \mathscr{B}$.

(d) Is $\mathcal{L}(\mathcal{H})=\cup\left\{\operatorname{Ran}\left(\delta_{A}\right)^{-}: A \in \mathcal{L}(\mathcal{H})\right\}$ [15]? Observe that if $P_{0}$ is a rank one orthogonal projection, the proof of Lemma 3 shows that $I+P_{0}$ is orthogonal to $\operatorname{Ran}\left(\delta_{A}\right)$ for all $A \in\left\{P_{0}\right\}^{\prime}$ in the sense that

$$
\operatorname{dist}\left\{I+P_{0}, \operatorname{Ran}\left(\delta_{A}\right)\right\}=\left\|I+P_{0}\right\|=2 \text {. }
$$

Question 4. Does $I+P_{0} \in \operatorname{Ran}\left(\delta_{A}\right)^{-}$for some $A \in \mathcal{L}(\mathcal{H})$ ?

\section{REFERENCES}

1. J. H. Anderson, Derivation ranges and the identity, Bull. Amer. Math. Soc. 79 (1973), 705-708. MR $48 \sharp 880$.

2. C. Apostol, C. Foias and D. Voiculescu, Some results on non-quasitriangular operators. IV, Rev. Roumaine Math. Pures Appl. 18 (1973), 487-514. MR 48 \#12109a.

3. J. Barria and D. A. Herrero, Closure of similarity orbits of Hilbert space operators. IV: Normal operators, J. London Math. Soc. (2) 17 (1978), 525-536.

4. A. Brown and C. Pearcy, Structure of commutators of operators, Ann. of Math. (2) 82 (1965), 112-127. MR 31 \$2612.

5. J. Dixmier, Les algèbres d'opérateurs dans l'espace hilbertien, Gauthier-Villars, Paris, 1957.

6. P. R. Halmos, A Hilbert space problem book, Van Nostrand, Princeton, N. J., 1967.

7. ___ Ten problems in Hilbert space, Bull. Amer. Math. Soc. 76 (1970), 887-933.

8. In I I I York, 1951.

9. D. A. Herrero, Quasisimilar operators with different spectra, Acta. Sci. Math. (Szeged) (to appear).

10. Norm limits of nilpotent operators and weighted spectra in non-separable Hilbert spaces, Rev. Un. Mat. Argentina 27 (1975), 83-105. 
11. Clausura de las órbitas de similaridad de operadores en espacios de Hilbert, Rev. Un. Mat. Argentina 27 (1976), 244-260.

12. _ Closure of similarity orbits of Hilbert space operators. II: Normal operators, J. London Math. Soc. (2) 13 (1976), 299-316.

13. D. A. Herrero and N. Salinas, Operators with disconnected spectra are dense, Bull. Amer. Math. Soc. 78 (1972), 525-526.

14. __ Analytically invariant and bi-invariant subspaces, Trans. Amer. Math. Soc. 173 (1973), 117-136.

15. H. W. Kim, On the unilateral shift and the norm closure of the range of a derivation, Math. Japon. 19 (1974), 251-256. MR 53 \$3783.

16. On compact operators in the weak closure of the range of a derivation, Proc. Amer. Math. Soc. 40 (1973), 482-486. MR $47 \sharp 7502$.

17. J. T. Marti, Introduction to the theory of bases, Springer-Verlag, New York, 1969.

18. D. Voiculescu, Norm-limits of algebraic operators, Rev. Roumaine Math. Pures Appl. 19 (1974), 371-378.

19. J. P. Williams, On the range of a derivation, Pacific J. Math. 38 (1971), 273-279.

Departamento de Matematicas, Instituto Venezolano de Investigaciones Cientificas, AP 1827, Caracas 101, Venezuela 